

météo (2)

WWW.FORUMETEOCLIMAT.COM fӨ우 FORUMETEOCLIMAT \#FIMC2021

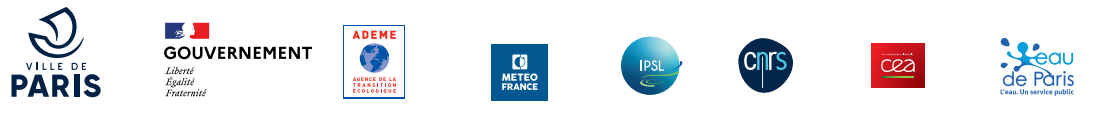
Schneider
Fondation

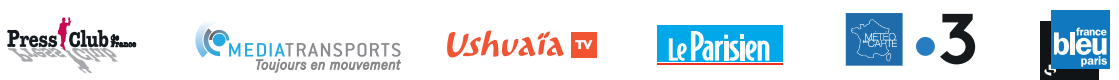




\section{Remise des prix Prud'homme 2019 et 2020}

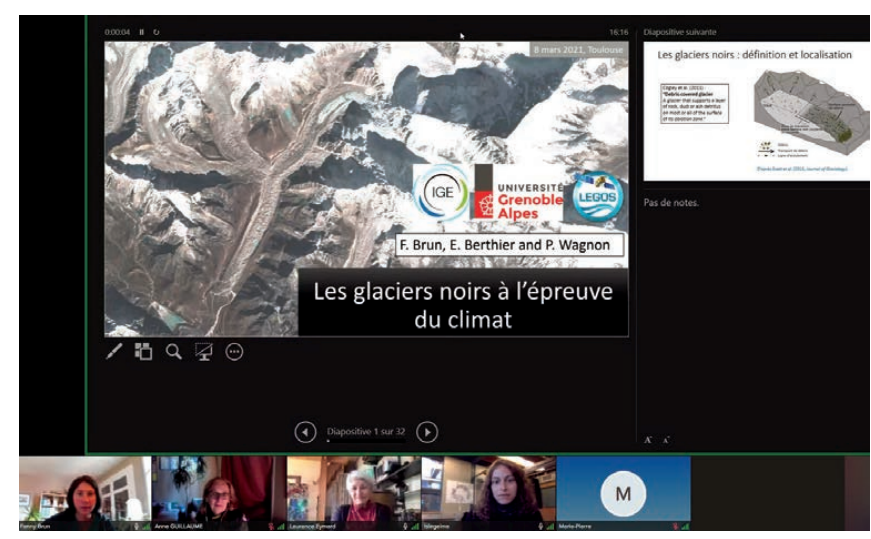

Figure 1. Extrait de l'exposé de Fanny Brun.

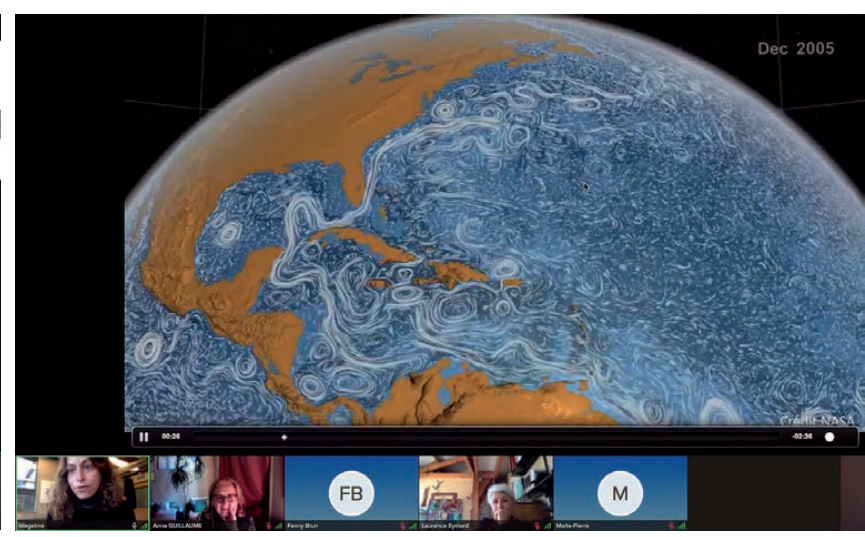

Figure 2. Extrait de l'exposé de Lia Siegelman. e Prix Prud'homme, créé en 1992, est attribué chaque année par Météo et Climat. Il récompense un jeune chercheur ayant soutenu une thèse de doctorat en météorologie, physique et chimie de l'atmosphère, paléoclimatologie, climatologie, y compris les aspects océanographiques.

Du fait de la crise sanitaire, la remise du prix 2019 prévue à Toulouse en mars 2020 n'avait pas pu avoir lieu, les ateliers de modélisation de l'atmosphère (AMA) ayant été annulés. Cette année, ces ateliers se sont déroulés du 8 au 12 mars 2021 en distanciel via la plateforme dédiée du CIC de Météo-France à Toulouse.

À cette occasion, Météo et Climat a été invitée à organiser le 8 mars la cérémonie de remise du prix 2019 décerné à Fanny Brun pour sa thèse intitulée « Les glaciers noirs à l'épreuve du climat » (figure 1) et celle du prix 2020, décerné à Lia Siegelman pour sa thèse intitulée "Impact climatique des fines échelles océaniques » (figure 2).

En présence d'Anne Guillaume, secrétaire générale de Météo et Climat et de Laurence Eymard, présidente du jury, les lauréates ont présenté un exposé sur leurs travaux respectifs devant les participants des AMA. Un temps d'échange leur a permis ensuite de répondre aux questions. Fanny Brun et Lia Siegelman ont été chaleureusement félicitées pour la qualité de leurs travaux et ont pu recevoir officiellement leur prix. Les articles rédigés par les lauréates ont été ou seront publiés dans La Météorologie.

Anne Guillaume, Morgane Daudier Météo et Climat

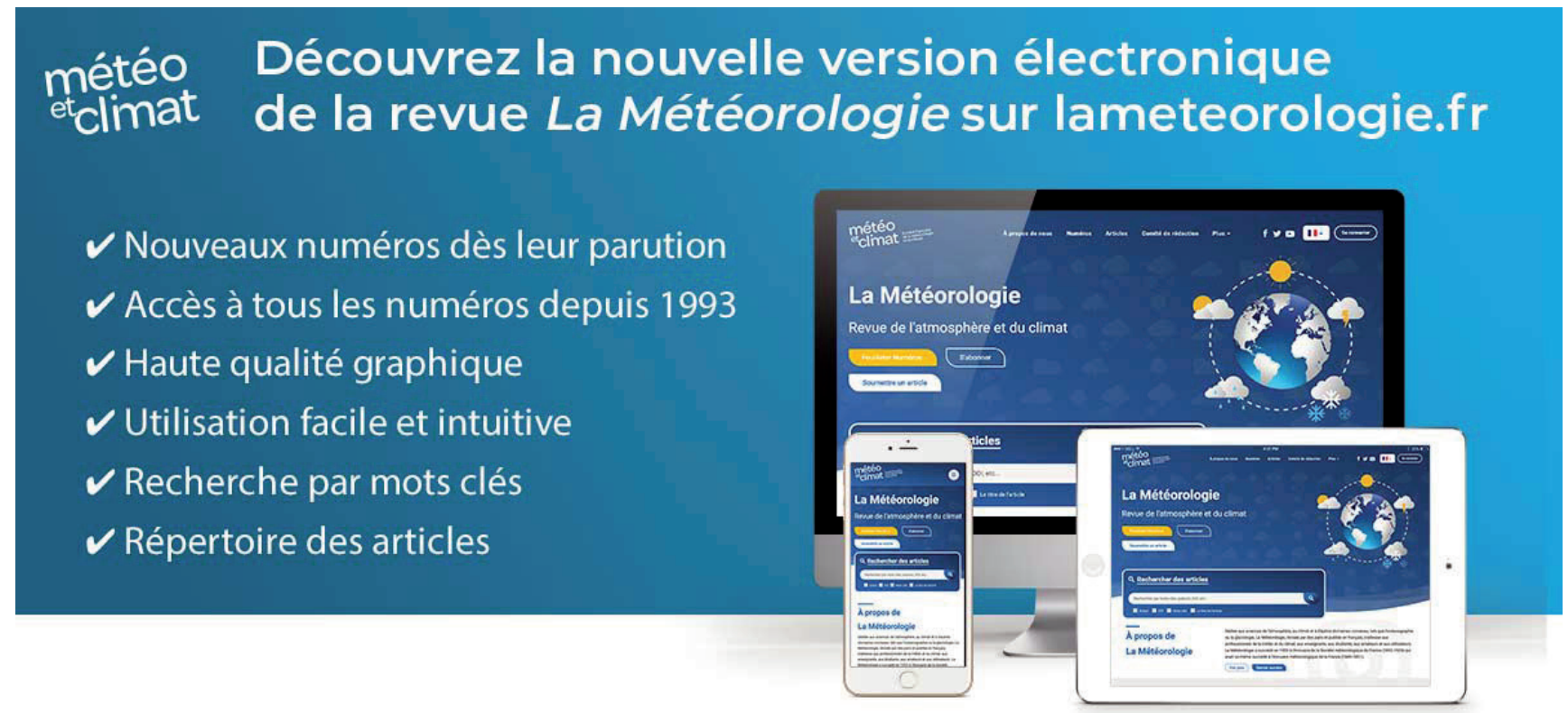




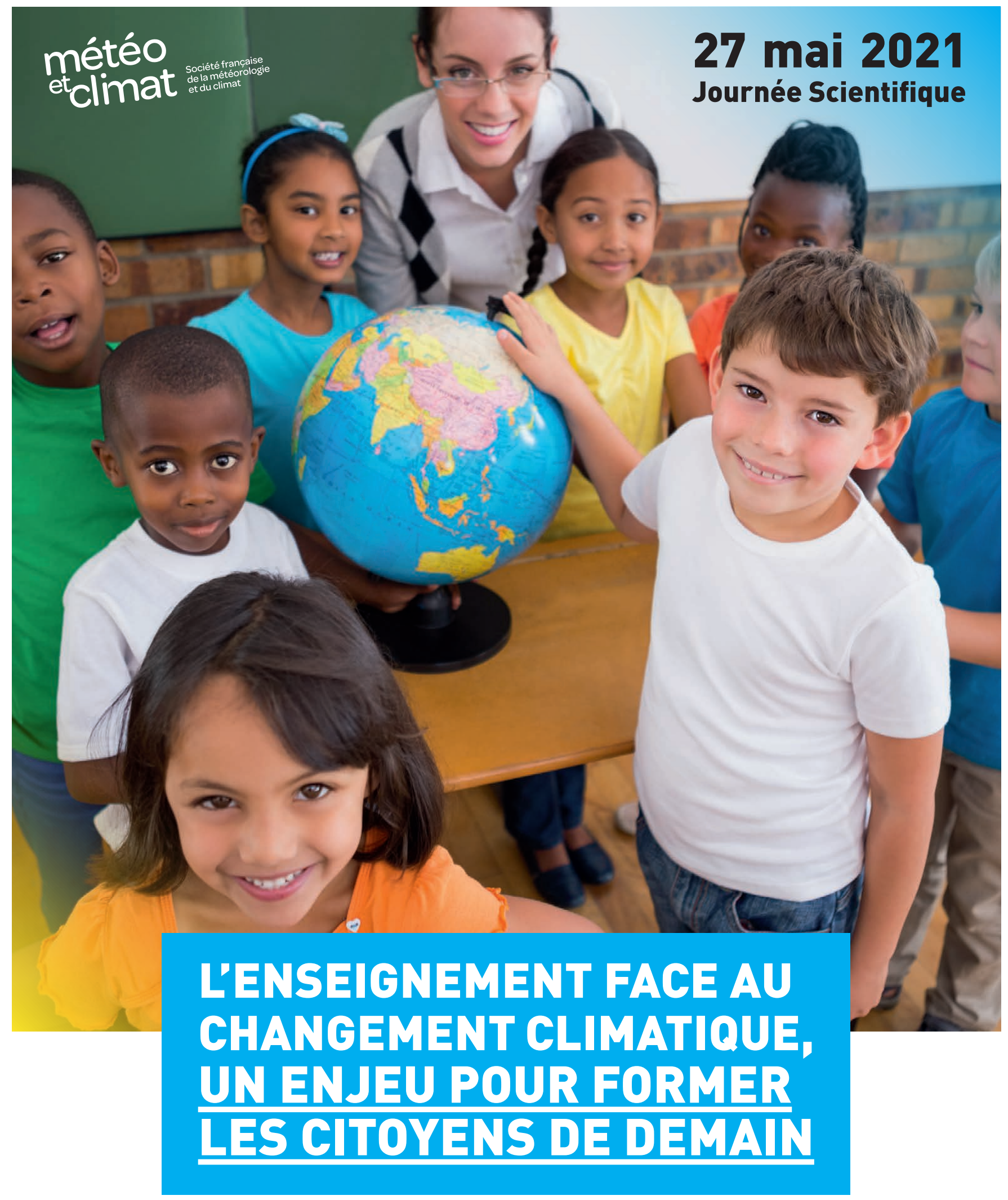

MUSÉUM NATIONAL D'HISTOIRE NATURELLE Amphithéâtre Verniquet, Paris $5^{\mathrm{e}}$

\section{Événement diffusé en direct sur internet • Inscriptions > www.meteoetclimat.fr}

\begin{tabular}{|c|c|c|c|c|c|c|}
\hline Parrainage & Partenair & fficiel & Partenaire & Ave & soutien de & \\
\hline 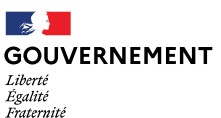 & 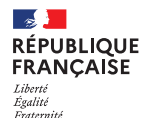 & $\begin{array}{c}\text { M } \\
\text { MEEIEO } \\
\text { FRACE }\end{array}$ & & $\begin{array}{l}\text { MUSËUM } \\
\text { NAL HIST } \\
\text { NATURELLE }\end{array}$ & OCE & \#JS2021 \\
\hline
\end{tabular}




\section{PRIX PERRIN DE BRICHAMBAUT APPEL A CANDIDATURES 2021}

\section{Un concours national sur la mêtéo et le climat à destination des écoles, les collèges et les lycées}

Le prix Perrin de Brichambaut a été créé en 1997 par l'association Météo et Climat afin d'honorer la mémoire de Christian Perrin de Brichambaut (1928-1995), ancien ingénieur à Météo-France et ancien président de Météo et Climat qui a beaucoup œuvré en faveur de la vulgarisation scientifique.

\section{RĖGLEMENT ET MODALITÉS}

Ce concours s'adresse aux écoles primaires, aux collèges et aux lycées qui réalisent au cours de l'année scolaire 2020-2021 un projet sur le thème de la météorologie, du climat et disciplines connexes explicitant un lien avec ces deux thématiques (développement durable, eau, environnement, énergie etc.). Les établissements scolaires étrangers sont autorisés à concourir dans la mesure où le projet soumis et ses annexes sont rédigés en langue française.

\section{JURY ET CRITĖRES D'APPRÉCIATION}

Les projets sont évalués par un jury composé de météorologistes et d'enseignants. La démarche scientifique, l'implication des élèves et la présentation des projets sont des critères majeurs d'appréciation.

\section{DOTATIONS}

$1^{\mathrm{er}}$ prix : $700 €-2^{\mathrm{e}}$ prix : $500 €-3^{\mathrm{e}}$ prix : $300 €$

\section{COMMENT CONCOURIR AU PRIX 2021 ?}

Il suffit de constituer un dossier comprenant

- Une lettre de candidature mentionnant les coordonnées postales de l'établissement, l'adresse e-mail et le numéro de téléphone de l'enseignant responsable du projet, le niveau de la classe participant au concours.

- L'intitulé du projet accompagné d'un descriptif détaillé (une page A4 maximum).

- Tous les supports permettant au jury d'apprécier le projet : dossier illustré, présentation ppt, vidéos, liens vers un site web...

\section{COMMENT ENVOYER LE DOSSIER?}

Par e-mail : info@meteoetclimat.fr

ou par courrier:

Météo et Climat

73, avenue de Paris, 94165 Saint-Mandé Cedex

\section{EXEMPLES DE PROJETS}

Lâchers de ballons sondes, observations et mesures en météorologie, création d'un site Internet, construction d'instruments...

\section{DATE LIMITE DU CONCOURS} 20 mai 2021

Date de réception de votre candidature par e-mail ou dans nos bureaux de Saint-Mandé.

Veuillez tenir compte des délais postaux.

\section{ATTRIBUTION DES PRIX}

La décision d'attribution des prix sera communiquée avant la fin de l'année scolaire et le $1^{\text {er }}$ prix sera remis dans l'établissement lauréat en juin 2021. 


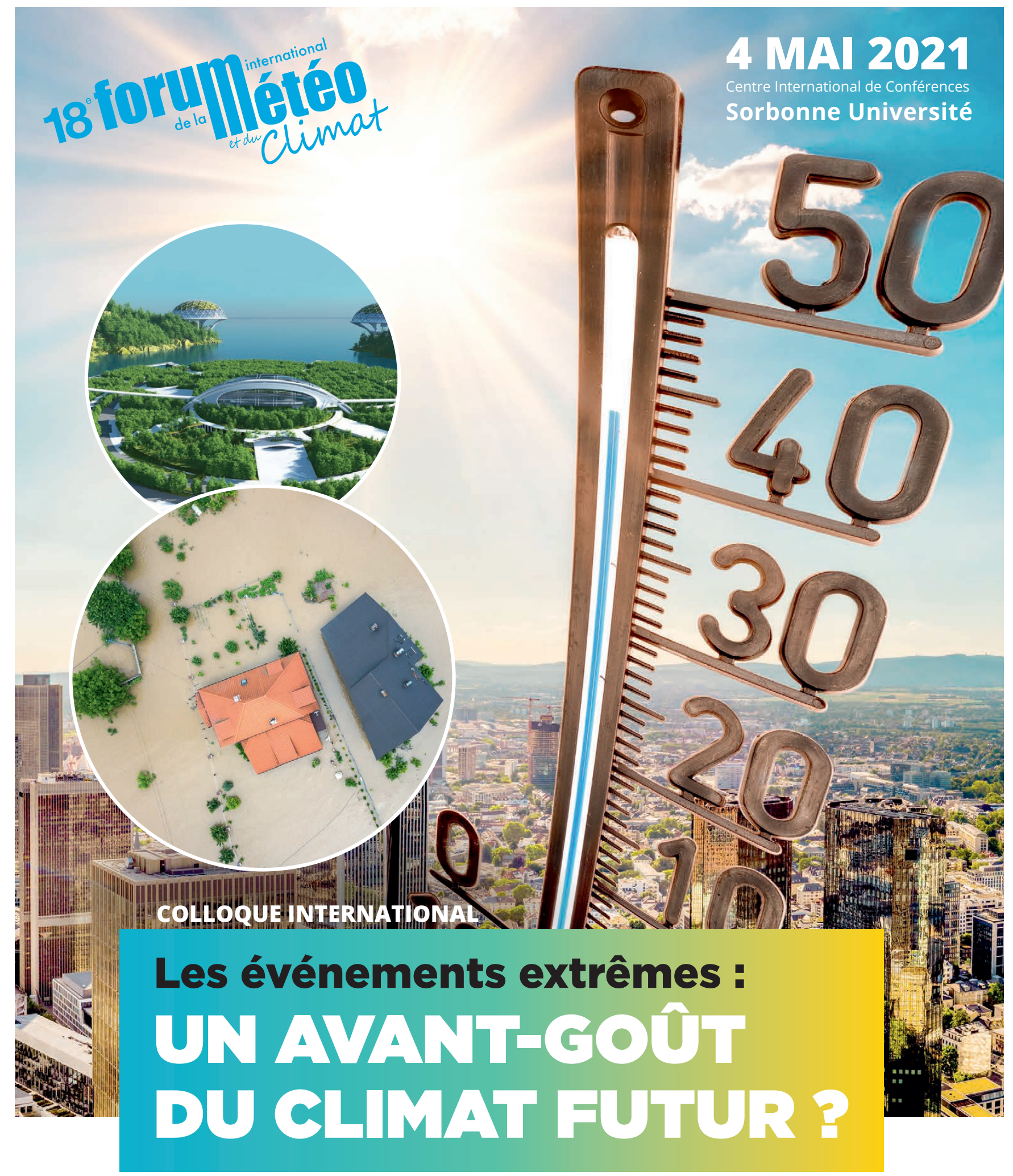

INSCRIPTIONS

WWW.FORUMETEOCLIMAT.COM PO? FORUMETEOCLIMAT \#FIMC2021 RETRANSMIS EN LIVESTREAM

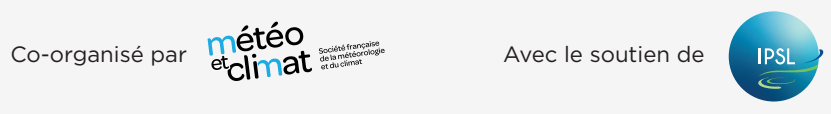

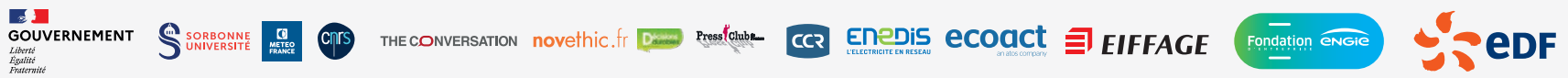

